\title{
Oxygen ion energization observed at high altitudes
}

\author{
M. Waara ${ }^{1}$, H. Nilsson ${ }^{1}$, G. Stenberg ${ }^{1}$, M. André ${ }^{2}$, H. Gunell ${ }^{3}$, and H. Rème ${ }^{4}$ \\ ${ }^{1}$ Swedish Institute of Space Physics, Kiruna, Sweden \\ ${ }^{2}$ Swedish Institute of Space Physics, Uppsala, Sweden \\ ${ }^{3}$ West Virginia University, Morgantown, USA \\ ${ }^{4}$ CESR, Toulouse, France
}

Received: 16 September 2009 - Revised: 28 January 2010 - Accepted: 22 March 2010 - Published: 8 April 2010

\begin{abstract}
We present a case study of significant heating (up to $8 \mathrm{keV}$ ) perpendicular to the geomagnetic field of outflowing oxygen ions at high altitude $\left(12 R_{\mathrm{E}}\right)$ above the polar cap. The shape of the distribution functions indicates that most of the heating occurs locally (within $0.2-0.4 R_{\mathrm{E}}$ in altitude). This is a clear example of local ion energization at much higher altitude than usually reported. In contrast to many events at lower altitudes, it is not likely that the locally observed wave fields can cause the observed ion energization. Also, it is not likely that the ions have drifted from some nearby energization region to the point of observation. This suggests that additional fundamentally different ion energization mechanisms are present at high altitudes. One possibility is that the magnetic moment of the ions is not conserved, resulting in slower outflow velocities and longer time for ion energization.
\end{abstract}

Keywords. Magnetospheric physics (Magnetosphereionosphere interactions)

\section{Introduction}

The ionospheric projection of the cusp/polar cap is an important source of outflowing oxygen ions. Ionospheric plasma may flow up from the ionosphere but at velocities which are low enough that the ions are still gravitationally bound (Nilsson et al., 1996). The initial upflow is caused by Joule heating of the ionospheric ions due to magnetospheric electric fields (Schunk et al., 1975), plasma heating due to particle precipitation and in particular ambipolar diffusion caused by effective heating of F-region electrons due to soft electron $(100 \mathrm{eV})$ precipitation typical of the cusp (Moore et al., 1999; Nilsson et al., 1998). For the ions to overcome gravity,

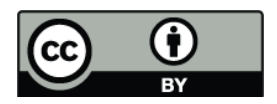

Correspondence to: M. Waara (martin.waara@irf.se) further acceleration is needed. The main energization mechanisms that have been known for ionospheric $\mathrm{O}^{+}$are ambipolar diffusion, wave-particle interaction, parallel potential drops and centrifugal acceleration (see André and Yau, 1997; Yau and André, 1997; Lotko, 2007). The energization needed for the particles to escape from the magnetosphere is even larger. Seki et al. (2002) discuss the further acceleration that is needed for the ions to escape into the magnetosheath rather than return to the Earth through the tail. In the cusp/polar cap, transverse heating is more common than field-aligned acceleration through a magnetic field aligned electric field. It is thus believed that transverse heating of ions is important for ion outflow and one of the probable explanations for transverse heating is wave-particle interaction. In a statistical study Norqvist et al. (1998) reported that at low altitudes $(1700 \mathrm{~km})$ most $\mathrm{O}^{+}$heating and ion outflow were caused by ion energization associated with low frequency broadband waves. Bouhram et al. (2004) investigated the altitude dependence of transversely heated $\mathrm{O}^{+}$distributions in the cusp region up to 6.5 Earth radii $\left(R_{\mathrm{E}}\right)$. They reported a saturation of transverse heating above radial distances $r>4.5 R_{\mathrm{E}}$. They suggested that such results may be interpreted as perpendicular wavelength effects in the wave-particle interaction. Nilsson et al. (2006) showed that the high altitude polar cap magnetosphere (6-12 $R_{\mathrm{E}}$ geocentric distance) is not just a passive transport region, and that a significant perpendicular energization at high altitudes was a likely explanation for the reported measurements. Arvelius et al. (2005) demonstrated that the statistical distribution of energetic ions $(1 \mathrm{keV}$ and above) in the 6-12 $R_{\mathrm{E}}$ geocentric distance interval was not consistent with just a velocity filter effect in combination with a low altitude heating source.

Nilsson et al. (2006) found that $\mathrm{O}^{+}$is significantly transversely energized in the high altitude region $\left(6-12 R_{\mathrm{E}}\right)$, indicated both by increasing perpendicular temperatures with altitude as well as by a tendency towards higher perpendicular than parallel temperature for the highest observed

Published by Copernicus Publications on behalf of the European Geosciences Union. 
temperatures. They did not further investigate the mechanism behind the heating. As the transversely accelerated ions subsequently move outward, their transverse energy is gradually converted to parallel energy by the mirror force. Such transversely heated and subsequently outflowing ions are known as "conics" due to their shape in velocity space (see André and Yau, 1997; Yau and André, 1997; Moore et al., 1999). A simple model (Chang et al., 1986), shows that gyroresonant heating by left-hand polarized waves, with wavelengths much larger than the ion gyroradius and at frequencies near the ion gyrofrequency, can be an efficient energizing mechanism (Norqvist et al., 1996). In such models the ions are heated by broadband waves. As the ions move up along the field lines, they interact with waves at a frequency that is in local resonance with them. The heating continues as long as the wave intensity remains strong. The calculation of the net increase of the perpendicular energy of the ions is based on mean values over many gyroperiods. Earlier studies at lower altitudes (up to $5 R_{\mathrm{E}}$ ), (Norqvist et al., 1996; André et al., 1998; Bouhram et al., 2003) found that only one to ten of percent of the observed spectral density around the oxygen gyrofrequency is needed to be in resonance with the ions to obtain the measured $\mathrm{O}^{+}$energies. No such study has been performed for the high altitude region.

The case study presented here is a follow up of the statistical study on high altitude oxygen ion energization reported by Nilsson et al. (2006). We investigate the longest heating event in our data set with clear conics at high altitude. The question we ask is if locally recorded waves can explain the strong heating we observed, and hence, if the heating mechanisms are likely to be the same at high altitudes as at low altitudes. We also determine how local the observed heating is. In particular in light of the apparent saturation of the heating at mid-altitudes attributed to finite wavelength effects (Bouhram et al., 2004) it is interesting to study the high altitude region where the gyro radius is even larger.

\section{Instrumentation}

We use data from the Cluster spacecraft. The four identical spacecraft are placed in a polar orbit $4 \times 19.6 R_{\mathrm{E}}$ (Escoubet et al., 2001). Data from three different instruments are used in this study. The Cluster Ion Spectrometry (CIS) is described in detail in Rème et al. (2001). The CIS package consists of two different instruments, a time-of-flight ion Composition Distribution Function (CODIF), which can resolve the major magnetospheric ions, and the Hot Ion Analyzer (HIA), which has no mass resolution but higher angular and energy resolution. By using a time-of-flight technique CODIF can resolve $\mathrm{H}^{+}, \mathrm{He}^{++}, \mathrm{He}^{+}$and $\mathrm{O}^{+}$. The angular resolution is $22.5^{\circ}$ and the energy coverage in the modes of interest to us is from $15 \mathrm{eV}$ per charge up to $38 \mathrm{keV}$ per charge. The energy range used in our event is from $40 \mathrm{eV}$ per charge up to $38 \mathrm{keV}$ per charge. The electric field and wave experiment (EFW) is designed to measure the electric field. EFW records two orthogonal electric field components in the satellite spin plane. In the event of interest the sampling rate is 25 samples/second (Gustafsson et al., 2001). Furthermore we use data from the Cluster fluxgate magnetometer (FGM). In our event the sampling rate is 22.4 samples/second (Balogh et al., 2001).

\section{Observations}

The event used in this study is recorded by the Cluster spacecraft. The event is selected from a larger data set including CIS CODIF data over a 3-year period (January to May in 2001 to 2003) of oxygen ions $\left(\mathrm{O}^{+}\right)$. This corresponds to orbits with apogee on the sunward side of the terminator plane. Our selected data set consists of clearly seen outflowing ions in the energy spectrograms. The data set is described by Nilsson et al. (2006). Our event (11 May 2002 at around 17:50-18:10 UT) was identified through visual inspection of perpendicular to parallel temperature ratio for the days in our data set. Figure 11 in Nilsson et al. (2006) shows that a high perpendicular to parallel temperature ratio exists and the temperature ratio often is high when the perpendicular temperature is high. High perpendicular to parallel temperature ratio is often a signature of local heating, as the mirror force will decrease the perpendicular temperature as the particle move up along the field line. We have selected an event where the perpendicular to parallel temperature ratio is higher than two. The high ratio remains for more than twenty minutes and is the longest period of such high ratio found in the data set. A convection velocity of $40 \mathrm{~km} / \mathrm{s}$ past the satellite during the 20 min corresponds to a length of $7.4 R_{\mathrm{E}}$ and the distance that spacecraft is traveling is $0.4 R_{\mathrm{E}}$. Events where the ratio is higher than two are common, but these events are normally just a few minutes long. A statistical distribution of the events is an expected follow up of this initial case study.

Figure 1 shows the energy spectrograms $\left(\mathrm{O}^{+}\right.$and $\left.\mathrm{H}^{+}\right)$, the ion temperatures and waves from spacecraft 1 , obtained at a geocentric distance of $12 R_{\mathrm{E}}$. For this time period the spacecraft starts in the magnetosheath and subsequently crosses the magnetopause (at 17:45 UT) into the mantle in the Southern Hemisphere. The top two panels, (a) and (b) show the $\mathrm{O}^{+}$and $\mathrm{H}^{+}$energy-time spectrogram, for the time around the heating event. The particle flux is integrated over all directions. Significant $\mathrm{O}^{+}$fluxes appear from 17:45 UT when the satellite moves from the magnetosheath into the mantle. The apparent $\mathrm{O}^{+}$fluxes before 17:45 are an instrumental artifact (Nilsson et al., 2006). We define the region as the mantle because the magnetosheath origin protons have mirrored at lower altitude and are moving outward, just as the oxygen ions (not shown). The parallel velocities of $\mathrm{H}^{+}$and $\mathrm{O}^{+}$are very close to the same (around $120 \mathrm{~km} / \mathrm{s}$ ) throughout our event, a situation which is typical in the high altitude polar cap (Nilsson et al., 2006). The thermal perpendicular 

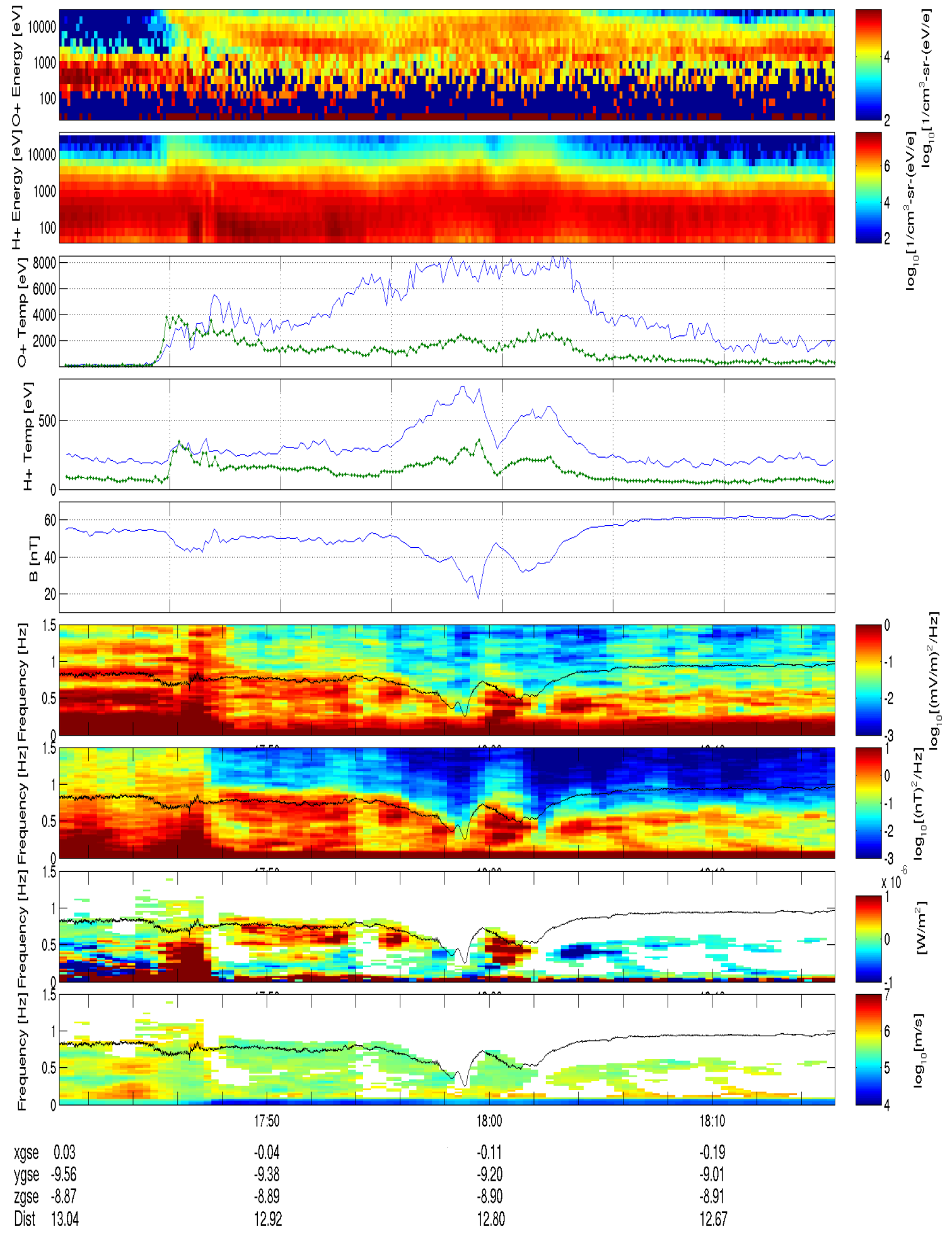

Fig. 1. Cluster spacecraft 1 data from 11 May 2002. Panels (a) and (b) show the $\mathrm{O}^{+}$and $\mathrm{H}^{+}$energy spectrograms, particle flux integrated over all directions. Panels (c) and (d) show the $\mathrm{O}^{+}$and $\mathrm{H}^{+}$temperature. (blue and green for perpendicular and parallel respectively). Panel (e) shows the total magnetic field. Panels (f) and (g) show the spectral density in the electric and the magnetic field. The black line in the panels is the proton gyrofrequency. Panel (h) shows the Poynting flux (blue for inward and red for outward Poynting flux). Panel (i) shows E/B. 

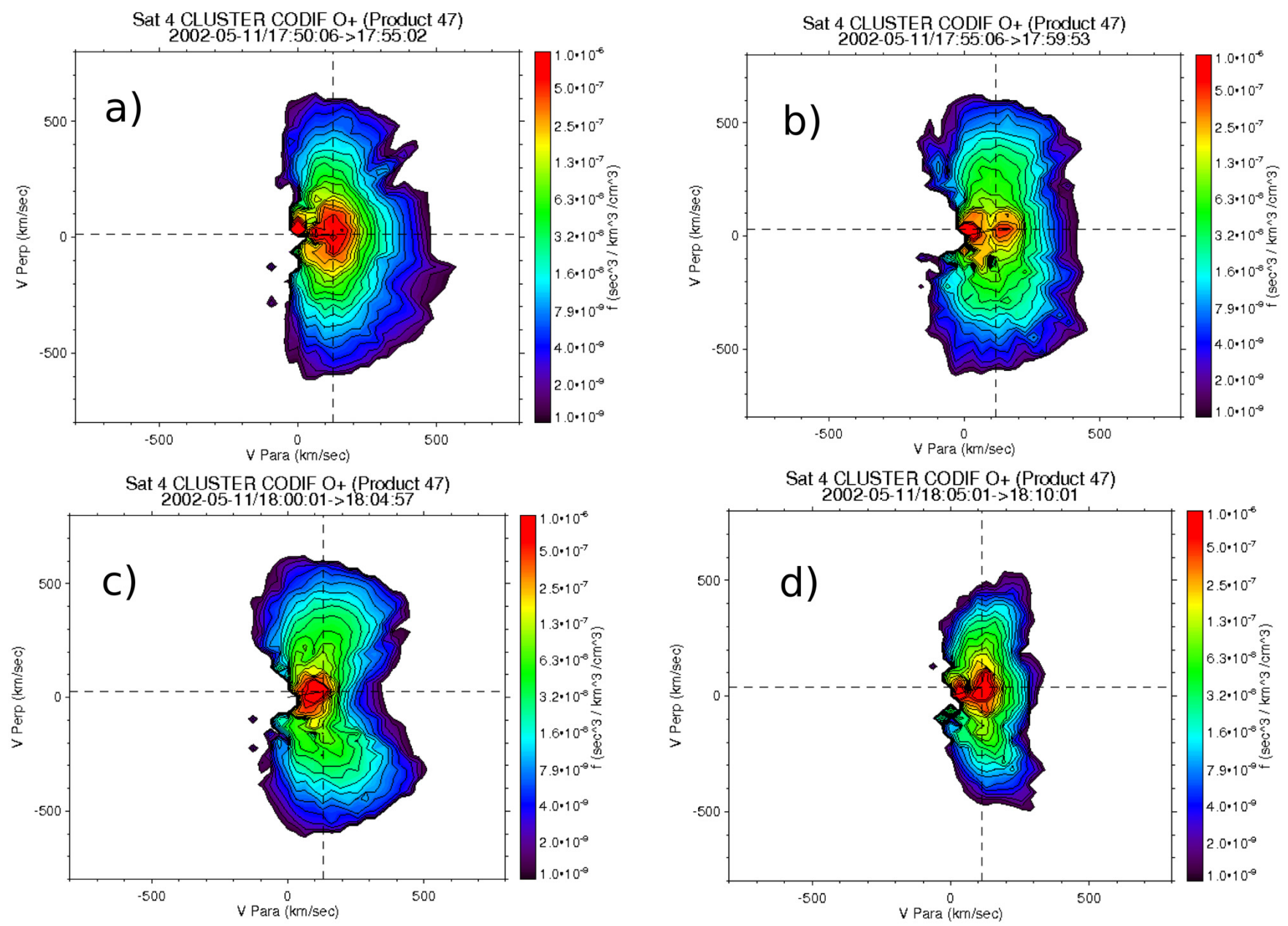

Fig. 2. Distribution functions of $\mathrm{O}^{+}$ions obtained during the event 11 May 2002 in a region of ion heating between, (a) 17:50 and 17:55, (b) 17:55 and 18:00, (c) 18:00 and 18:05, (d) 18:05 and 18:10.

velocity are also the same for both oxygen ions and protons, around $310 \mathrm{~km} / \mathrm{s}$. Hence the energy is higher for the oxygen population than for the proton population. The panels (c) and (d) show the $\mathrm{O}^{+}$and $\mathrm{H}^{+}$temperature. (blue and green for perpendicular and parallel, respectively). The high perpendicular to parallel ratio for the oxygen temperature starts a few minutes after the spacecraft entered the mantle. Just inside the magnetopause the parallel temperature of $\mathrm{O}^{+}$is significantly larger than the perpendicular temperature. In the interval between 17:50 and 18:10 the high perpendicular to parallel temperature ratio is continuous although the perpendicular temperature is decreasing. The perpendicular temperature is much higher (up to $8 \mathrm{keV}$ ) in our event than events reported at lower altitudes, e.g. up to $1 \mathrm{keV}$ at $5 R_{\mathrm{E}}$, (Bouhram et al., 2004). The total magnetic field in panel (e) shows two cavities, one before 18:00 and one after. The magnetic cavities are associated with an increase in plasma pressure due to higher $\mathrm{H}^{+}$temperature. Panels (f) and (g) show the power spectral density in the electric and the magnetic field.
The black line in the panels is the proton gyrofrequency. Analysis of the wave data indicates that we have left-hand polarized electromagnetic waves under the proton gyrofrequency. A hodogram (not shown) reveals that the waves are left-hand polarized. After passing through the magnetopause at 17:45 the spectral density is significant only below the proton gyrofrequency for both the electric field and the magnetic field. After 18:00 the spectral density is significant only below around half of the proton gyrofrequency. The Poynting flux in panel (h) shows that the energy flux in the frequency just below the proton gyrofrequency is along the magnetic field line (i.e. outward, apparently coming from below) and at the lower frequencies it is antiparallel to the magnetic field lines between 17:50 and 18:05. After 18:05 the Poynting flux is antiparallel to the magnetic field line. In panel (i) we shown $\mathrm{E} / \mathrm{B}$ which is an estimate of the phase velocity. It is a few hundred $\mathrm{km} / \mathrm{s}$ which is close to the Alfvén velocity, and the observations are consistent with Alfvén waves. 
Four sample distribution functions obtained during the heating event are presented in Fig. 2. The distribution functions are averages over four consecutive $5 \mathrm{~min}$ intervals between 17:50 and 18:10 UT. The upper left panel, (a) shows a distribution with $T_{\perp} / T_{\|}>1$, but with no signs of conic formation (folding due to mirror force). The distribution function indicates that we have heating at or just below the spacecraft. In the next two panels, (b) and (c) the distribution is folded up 10 to $15^{\circ}$ from the perpendicular direction. The folding indicates that the population have been drifting upward some distance since the heating took place. The apex angle is used to estimate the extension of the heating region. The apex angle in the lower left panel is $15^{\circ}$ and in a dipole geomagnetic field it corresponds to a heating region of $0.2 R_{\mathrm{E}}$. Most of the particles are outgoing particles but in the (b) and (c) panels there are some particles with negative parallel velocity, i.e. downgoing particles. Perpendicular heating over extended altitudes in combination with the mirror force only give rise to outgoing particles. In addition to the perpendicular heating there must exist some parallel acceleration/heating, significant pitch angle scatter or reflection further along the field line. In the distribution shown in panel (d) the temperature is lower.

The electric field spectral densities for the time intervals corresponding to the distribution functions shown in Fig. 2 are shown in Fig. 3 and panel (f) in Fig. 1. All four spectra show a strong low frequency component in the vicinity of the oxygen gyrofrequency. The spectral density is nearly constant around the $\mathrm{O}^{+}$gyrofrequency for the time period between 17:50 and 18:10. There are small changes with time (a few tens of percent) in the electric field spectral density at around half the proton gyrofrequency. In between half the proton gyrofrequency and the proton gyro gyrofrequency the wave power decrease with time after 18:02, particularly clear in the color spectrogram, panel (h) in Fig. 1. Regardless of the different shape of the simultaneous measured distribution function the observed spectral density is about $2\left[(\mathrm{mV} / \mathrm{m})^{2} / \mathrm{Hz}\right]$ at the oxygen gyrofrequency. The very good correspondence between the upper limit of the wave frequencies and the proton gyrofrequency indicates that we do not have significant Doppler shift of the waves, at least around the proton gyro frequency.

\subsection{Ion heating}

We have used the model described by Chang et al. (1986) to calculate the heating of $\mathrm{O}^{+}$from the waves. This model assumes a broadband spectrum of waves around the gyrofrequency. The heating rate is given by

$\frac{d w}{d t}=S_{L} \frac{q^{2}}{2 m}$

where $q$ and $m$ are the charge and the mass of the ion and $S_{L}$ is the power spectral density in the electric field at the $\mathrm{O}^{+}$gyro frequency due to left-hand polarized waves. We
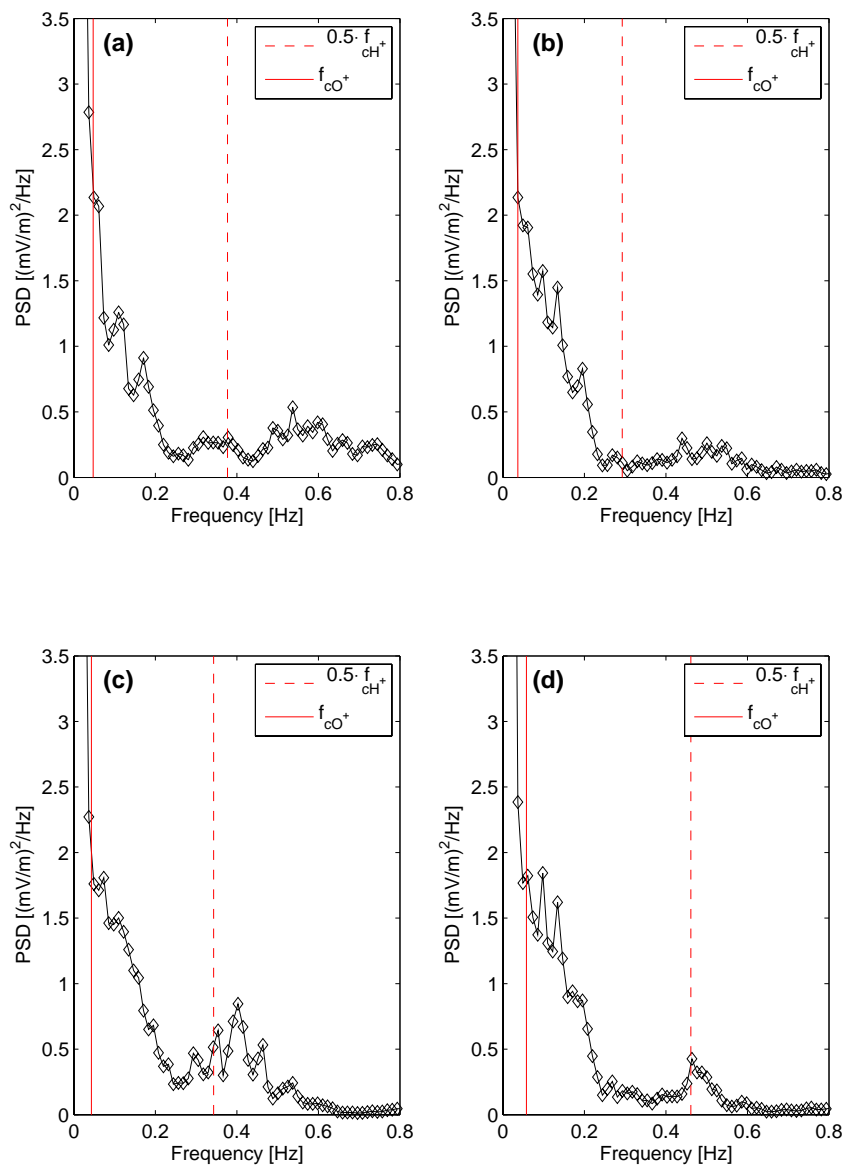

Fig. 3. The power spectral density for the electric field observed during the event (see Fig. 1). The vertical solid line and dashed line indicate the oxygen gyrofrequency and half of the proton gyrofrequency respectively. The time span in panel: (a) is 17:50-17:55, (b) is 17:55-18:00, (c) is 18:00-18:05 and (d) is 18:05-18:10.

follow the particle in a prescribed dipolar magnetic field under the influence of the mirror force and a constant heating rate. We started the test particle calculation with the measured values and ran backwards in time until the parallel velocity was zero. The measured values we have used as initial values in the test particle calculation are, perpendicular velocity of $310 \mathrm{~km} / \mathrm{s}$, parallel velocity of $120 \mathrm{~km} / \mathrm{s}$ and $2\left[(\mathrm{mV} / \mathrm{m})^{2} / \mathrm{Hz}\right]$ spectral density. The backward test particle calculation presented in Table 1 shows that the particles must have started $0.4 R_{\mathrm{E}}$ below the spacecraft with no parallel velocity and $9000 \mathrm{eV}$ in perpendicular energy to get the measured values. The distance we got from the test particle calculation, $\left(0.4 R_{\mathrm{E}}\right)$ is twice the distance that we got from the apex angle and dipole field. The distance from the test particle calculation is the maximal distance the distribution have traveled along the magnetic field lines if the spectral density is constant $\left(2\left[(\mathrm{mV} / \mathrm{m})^{2} / \mathrm{Hz}\right]\right)$. The heating rate for the test particle calculation is $6 \mathrm{eV} / \mathrm{s}$ for oxygen. To get 
Table 1. Summary of the of backwards test particle calculation. The left column in the table shows the test particle calculation from the measured values (stop) down to the mirror point (start) for $\mathrm{O}^{+}$, and the right column shows the test particle calculation for $\mathrm{H}^{+}$.

\begin{tabular}{lcc}
\hline & $\mathrm{O}^{+}$ & $\mathrm{H}^{+}$ \\
\hline$V_{\|}$start $[\mathrm{km} / \mathrm{s}]$ & 0 & 0 \\
$E_{\perp}$ start $[\mathrm{eV}]$ & 8961 & 15 \\
length/time $\left[R_{\mathrm{E}} / \mathrm{s}\right]$ & $0.41 / 42$ & $0.4 / 38$ \\
Spectral density $\left[(\mathrm{mV} / \mathrm{m})^{2} / \mathrm{Hz}\right]$ & 2 & 0.35 \\
$V_{\|}$stop $[\mathrm{km} / \mathrm{s}]$ & 120 & 63 \\
$E_{\perp}$ stop $[\mathrm{eV}]$ & 8000 & 600 \\
Heating rate $[\mathrm{eV} / \mathrm{s}]$ & 6 & 16.8 \\
\hline
\end{tabular}

the observed perpendicular oxygen temperature with the observed field we must start with $9000 \mathrm{eV}$. Thus, the heating of the oxygen can not be explained with the observed spectral density even when we assume that $100 \%$ of the observed spectral density is effective in heating the ions.

The protons also show an increase in temperature in the same manner as $\mathrm{O}^{+}$. We therefore run our model calculation for protons as well. The backward test particle calculation gives almost the same mirror point for protons and $\mathrm{O}^{+}$but when the protons mirror the perpendicular energy is only $15 \mathrm{eV}$. If they were cold ionospheric origin protons this could possibly be reasonable, but it is too low for magnetosheath origin ions. As we interpret the $\mathrm{H}^{+}$ion population as mirrored ions of magnetosheath origin, the heating of the $\mathrm{H}^{+}$ions is likely less than in our model. We used $100 \%$ of the spectral density for protons of $0.35\left[(\mathrm{mV} / \mathrm{m})^{2} / \mathrm{Hz}\right]$ and the heating rate was $16.8 \mathrm{eV} / \mathrm{s}$. The heating of $\mathrm{H}^{+}$ions is thus consistent with the cyclotron resonance model, but if the protons had a significantly higher perpendicular temperature than $15 \mathrm{eV}$ when it mirrored, only a part of the observed spectral density was efficient in heating the protons, just as for lower altitudes. The results of the test particle calculation are summarized in Table 1.

To investigate if the wave heating could work if the waves are in perfect resonance with the particles for, as an example, an entire gyroperiod, we perform a second test particle calculation. The second test particle calculation is made only for oxygen and we have assumed full resonance for one gyroperiod and used an amplitude of the E-field of $1 \mathrm{mV} / \mathrm{m}$. The amplitude of the measured E-field is shown in Fig. 4. We have used $\Delta v=\left(q E_{\perp} / m\right) \Delta t$ to calculate the energy change from the electric field. The heating rate we get from the model is between 230 and $258 \mathrm{eV} / \mathrm{s}$. This maximum test particle calculation is presented in Table 2 .

The extension of the heating area, $\left(0.3 R_{\mathrm{E}}\right)$ is consistent with the earlier estimations. In this event the perpendicular energy of ions increases from $3.8 \mathrm{keV}$ at the mirror point to $8 \mathrm{keV}$ in a reasonable time/distance and the energy gain is of the right order of magnitude. However, this should be an overestimate of any real heating mechanism.
Table 2. Backward test particle calculation. We have used $\Delta v=$ $\left(q E_{\perp} / m\right) \Delta t$ to calculate the energy change.

\begin{tabular}{lc}
\hline & $\mathrm{O}^{+}$ \\
\hline$V_{\|}$start $[\mathrm{km} / \mathrm{s}]$ & 86 \\
$E_{\perp}$ start $[\mathrm{eV}]$ & 3861 \\
length/time $\left[R_{\mathrm{E}} / \mathrm{s}\right]$ & $0.3 / 18$ \\
E-field $[(\mathrm{mV} / \mathrm{m})]$ & 1 \\
$V_{\|}$stop $[\mathrm{km} / \mathrm{s}]$ & 120 \\
$E_{\perp}$ stop $[\mathrm{eV}]$ & 8000 \\
Heating rate $[\mathrm{eV} / \mathrm{s}]$ & $230-258$ \\
\hline
\end{tabular}

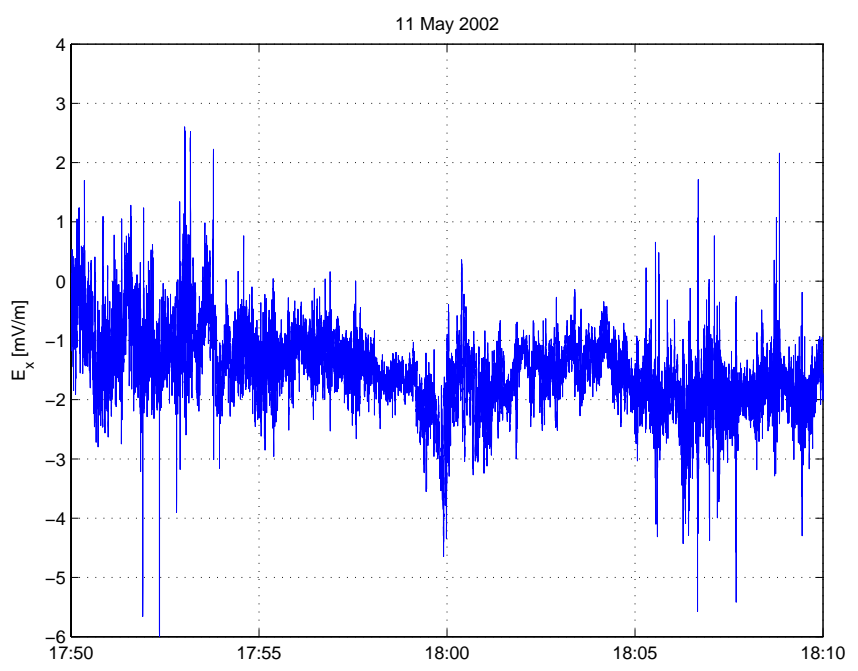

Fig. 4. The time series of the wave electric field.

It is not entirely obvious that the magnetic moment is conserved as the parallel velocity is quite high $(120 \mathrm{~km} / \mathrm{s})$ and the $\mathrm{O}^{+}$gyroperiod is very long $(18 \mathrm{~s})$ so that the magnetic field may possibly not vary as slowly as assumed in the derivation of the conservation of the magnetic moment. This would strongly affect our results as all theories assume the magnetic moment to be conserved in the absence of heating. In a simple test run, ion trajectories were computed by integrating the Lorentz force using Boris' algorithm (see for example, Hockney and Eastwood, 1988), and assuming $\mathbf{E}=0$ shows that in a static Tsyganenko magnetic field model (Tsyganenko, 1989) we should expect the magnetic moment to be conserved at these parallel velocities and gyroperiods.

For some high altitude sudden heating events, the temperature in the heating region is isotropic and not conic shaped, as in the event reported in Nilsson et al. (2004). This indicates that heating processes may significantly affect also the parallel component of the ion velocity. Furthermore the close coupling between the parallel bulk velocities of $\mathrm{H}^{+}$and $\mathrm{O}^{+}$typically seen in the high altitude mantle region (Nilsson et al., 2006) as well as in this particular event indicates that 
parallel velocity of $\mathrm{O}^{+}$is affected by more factors than the mirror force.

\section{Discussion}

The statistical study of Nilsson et al. (2006) showed significant transverse energization of ions at high altitudes, indicated both by high perpendicular temperatures for low magnetic field values as well as by a tendency towards higher perpendicular than parallel temperature on distributions for the highest observed temperatures. The mechanisms behind the heating were not determined.

In our event the particles appear locally heated; the perpendicular temperature is high; and a high ratio between perpendicular and parallel temperature remains for a long time, $20 \mathrm{~min}$. The power spectral density in the electric field is around $2(\mathrm{mV} / \mathrm{m})^{2} / \mathrm{Hz}$ at the oxygen gyrofrequency; the extension of the heating region calculated from the apex angle is $0.2 R_{\mathrm{E}}$; the amplitude of the electric field is on the order of $1 \mathrm{mV} / \mathrm{m}$; and we need a heating of the order of some $\mathrm{keV}$. What time span is available for the heating?

Using the $V_{\|}$start and $V_{\|}$stop from Table 1 gives an average parallel velocity of about $60 \mathrm{~km} / \mathrm{s}$. The small apex angle in our distribution functions (up to $15^{\circ}$ ) and an average parallel velocity of about $60 \mathrm{~km} / \mathrm{s}$ indicates that particles have been heated within $20 \mathrm{~s}$ and $0.2 R_{\mathrm{E}}$ below the spacecraft. Another potential explanation for the high perpendicular temperature is that the particles are heated above the spacecraft and are mirrored just below the spacecraft. It is unlikely for the spacecraft to spend 20 min closely above the the mirror point, while only observing the upward, mirrored, component and not the downward incident ions. Another mechanism that could be responsible for observed heating is centrifugal acceleration (Cladis, 1986). We have calculated the centrifugal acceleration as in Nilsson et al. (2008) Assuming for simplicity that centrifugal acceleration varies only with altitude, we have summed up the centrifugal acceleration, as observed along the orbit, from the lowest altitude at 19:00 UT up to the highest altitude we use (17:45 UT). The cumulative sum of the centrifugal acceleration relative lowest altitude is only $1.5 \mathrm{~km} / \mathrm{s}$.

The length drifting past the spacecraft during the heating event by convection is $7.4 R_{\mathrm{E}}$ and the drift is at about $45^{\circ}$ angle relative to the spacecraft trajectory, with the component along the trajectory directed away from the magnetopause crossing at 17:45. The magnetopause does not pass the spacecraft after the encounter at 17:45. If we do not have reconnection, the magnetopause should drift together with the plasma and could thus drift past the spacecraft. This does not happen, and it is thus not likely that we are close to the magnetopause throughout the intense heating interval. Because of the fact that we have a significant and consistent drift along the satellite orbit the length of the heating area is most likely at least of the same size as the distance that the spacecraft is travelling $\left(0.4 R_{\mathrm{E}}\right)$.

Is it possible that heating occur only at the magnetopause? If the distance from the boundary is less than a gyroradius we would see gyro anisotropy in the ion distribution function. Due to the fact that we can see particles coming from all direction in the distribution functions the size of the heating area must be at least a few gyroradii. We have previously derived that particles have been heated within $20 \mathrm{~s}$ and $0.2 R_{\mathrm{E}}$ below the spacecraft. This, together with a perpendicular drift velocity of about $40 \mathrm{~km} / \mathrm{s}$ we find that if heating occur only at the magnetopause, large enough perpendicular oxygen temperatures can be obtained only within less than a gyroradius (around $800 \mathrm{~km}$ ) from the magnetopause. The heating area can be close to the magnetopause but it is not possible that the particles have been heated at the magnetopause only. As a further argument against heating only at the magnetopause, the ions are observed inside the magnetopause in the mantle region. It is difficult to see how such field lines can have been adjacent to the magnetopause at lower altitudes at an earlier time.

Does the shape of the distribution function necessarily imply local heating? Barghouthi (2008) showed that the shape of conic distributions such as those shown in Fig. 2 are consistent with remote, not local, heating. In a numerical cyclotron resonance simulation of $\mathrm{O}^{+}$ion velocity distributions conics can appear at $2 R_{\mathrm{E}}$ and still be conically shaped up to $9.6 R_{\mathrm{E}}$. The shape of the distribution functions in their infinite wavelength event is similar to the shape that we see in our event. Although a similar shape of the distribution function as we have is reproduced by simulations, the parallel velocity would be too high when the particles reach altitude of our observations. This is not dependent on the details of the heating mechanism, only on the effect of the mirror force. Therefore the shape of the distribution function in combination with the relatively low parallel bulk velocity, as compared to the perpendicular thermal velocity, indeed indicates local heating.

Fritz et al. (2003) have observed large diamagnetic cavities in the dayside high altitude cusp region. These cavities were associated with energetic ions. In our study there are two cavities in the magnetic field but the heating of oxygen is not affected by the low magnetic field region. Most of the heating occurs before the cavities are observed, when the magnetic field is around $60 \mathrm{nT}$. The oxygen temperature is fairly constant in the low magnetic field region and starts to decrease after the observation of the magnetic cavities.

There is no clear correlation between heating of the particles and the wave activity. From the distribution function it is clear that most of the heating appears in the beginning of the event and less heating in the second half of the event. The maximal spectral density at relevant frequencies are similar during the whole event. The spectral density is too small to explain the observed heating using conventional theory used at lower altitudes, e.g. observations obtained by Freja 
satellite at altitudes around $1700 \mathrm{~km}$ (Norqvist et al., 1996; André et al., 1998) or by Interball-2 satellite at altitudes up to $4 R_{\mathrm{E}}$ (Bouhram et al., 2003). Even when we assume a coherent E-field at the $\mathrm{O}^{+}$gyrofrequency, rather than realistic incoherent broadband waves, the resulting heating is not enough. The observed apex angle of the ion distributions and hence a limited extent of the ion heating region along the geomagnetic field line give a limited time for the heating mechanism to operate on each ion.

The clearest difference as compared to low altitudes $(1700 \mathrm{~km})$ are the weak magnetic field and high energy of the particles and consequent large oxygen ion gyroradius and long gyroperiod. At midaltitude, $5 R_{\mathrm{E}}$ finite gyroradii effects have been suggested as a mechanism to suppress heating. Bouhram et al. (2004) reported a saturation of transverse heating processes and the limited $\mathrm{O}^{+}$gyroradii may be interpreted as the consequence of finite wavelength effects in the wave-particle interactions. At still higher altitude, $12 R_{\mathrm{E}}$ in our example, the heating must be very efficient to explain both the perpendicular temperature and the relatively low resulting parallel velocity.

The low spectral density of the observed electric field as compared to what are needed in our test particle calculation implies that it does not matter exactly what type of wave particle interaction we have (i.e. stochastic heating) (Chaston et al., 2004) or gyroresonance (Chang et al., 1986). The ions must be in coherent interaction with an accelerating electric field for the order of one gyro period of around $20 \mathrm{~s}$. The DC electric field could possibly play a role in accelerating particles with large gyro radii, if there are fields of DC character on the ion gyro time scale, which have appreciable spatial changes within the ion gyro radius length scale. But the DC field is about $2-3 \mathrm{mV} / \mathrm{m}$, i.e. not much more than the wave field. Another possible explanation is large sporadic E-field outside the orbit of the Cluster spacecraft. It is unlikely that such electric fields have heated the particles in our event. They would have to occur rather close to the observation point, and would have to be large and exist for a long time. If that were the case, these fields should be observed during our $20 \mathrm{~min}$ interval, but they are not.

The spectral density is nearly constant around $\mathrm{O}^{+}$gyrofrequency during the whole event but the observed heating of particles occurs only when the Poynting flux is outward. Outward moving waves come from a region with larger magnetic field or higher gyrofrequency. Waves exist up to the proton gyrofrequency and waves with downgoing Poynting flux are seen only well below the proton gyrofrequency. If the same is true for waves around the oxygen gyrofrequency, we would expect strong wave-particle interaction mainly for outgoing waves. A better resolution at the oxygen gyrofrequency requires longer sampling time. The fact that heating of particles only occurs when the Poynting flux is outward and significant Poynting flux exist close to the gyrofrequency is consistent with wave heating of the particles.
Are there other alternative explanations? In the test particle calculation we assume that the magnetic moment is conserved. If the magnetic moment is not conserved the particles have more time available for heating, they may have started further down in the magnetosphere and are not folded up and do not reach parallel velocities that are too high; this puts a limit on heating over extended altitude intervals. However, we have made a simple calculation and in this showed that the magnetic moment is conserved in a static magnetic field for the velocities we observe.

The thermal velocity is the same for both oxygen ions and protons, around $310 \mathrm{~km} / \mathrm{s}$. The same parallel velocities indicate that more energy is fed into the $\mathrm{O}^{+}$population than into the $\mathrm{H}^{+}$population. This does not agree with cyclotron resonance calculation for our case. It rather implies that the close coupling between the $\mathrm{H}^{+}$and $\mathrm{O}^{+}$ion populations manifested in the close to same parallel bulk velocities (Nilsson et al., 2006) is also an integral part of the high altitude perpendicular heating of $\mathrm{O}^{+}$.

\section{Conclusions}

We have studied significant perpendicular heating of outflowing oxygen ions at high altitude above the polar cap using Cluster/CIS data. The small apex angle and the relatively small parallel velocity indicate that most of the heating is local, within $0.2-0.4 R_{\mathrm{E}}$. We see the heating for $20 \mathrm{~min}$ inside the cusp/mantle. We observe large regions of clear $\mathrm{O}^{+}$conics at high altitude, $12 R_{\mathrm{E}}$. These conics have large energies (high temperatures), up to $8 \mathrm{keV}$ in the perpendicular direction. This is much higher than energies of conics at lower altitudes, e.g. up to $1 \mathrm{keV}$ at $5 R_{\mathrm{E}}$ (Bouhram et al., 2004).

These observations raise the question of how the particles are energized. First we note that electromagnetic left-hand polarized waves are observed at the same time as the ion conics. However, we see no obvious correlation between the perpendicular $\mathrm{O}^{+}$temperature and the waves. There are also magnetic cavities inside the ion heating region, but we see no correlation between the $\mathrm{O}^{+}$temperature and these either.

To make a quantitative estimate of the maximum possible ion heating by the observed electric field we have tested a few scenarios. First we assume that all the electric field spectral density at the oxygen gyro frequency is due to incoherent left-hand polarized waves. We find that the obtained heating is far below what is needed to explain the observations (by a factor of 100). If we instead use the extreme assumption that all the observed electric field wave amplitude is due to a coherent electric field at the oxygen gyro frequency, the obtained heating is still not enough to explain the observations.

Another possibility is that the ions are heated when the flux tube was close to the magnetopause and have drifted perpendicularly to the region where they are observed. Assuming a large enough potential drop at the magnetopause observed at the beginning of the ion heating event, and using 
the observed $\boldsymbol{E} \times \boldsymbol{B}$-drift into the ion heating region, such a combination of a static potential drop and drift cannot explain the observed ion temperatures. The spacecraft travels perpendicularly to the magnetopause boundary and hence, the particle distribution would change considerably during the 20 min we observe strong heating. The heating area can be close to the magnetopause but it is not likely that the particles have been heated at the magnetopause. Given the size of the heating region the area where heating is observed must be at least a few gyroradii wide.

In summary, we observe strong perpendicular oxygen heating at high altitudes but have difficulties in finding the heating mechanism. A reasonable suggestion is that more time must be available for heating. This could happen if the magnetic moment is not conserved, but a simple test particle calculation indicated that the magnetic moment is conserved. It therefore suggests that a new heating mechanism is operating. This is likely connected to the observation that $\mathrm{H}^{+}$and $\mathrm{O}^{+}$parallel velocities are close to the same, which in turn indicates that much more energy is fed into the $\mathrm{O}^{+}$population than into the $\mathrm{H}^{+}$population.

Acknowledgements. M. Waara was financed by the Swedish National Graduate School of Space Technology. H. Nilsson was supported by the Swedish Research Council. M. André and G. Stenberg were supported by the National Space Board of Sweden. We also thank the CIS, EFW and FGM instrument teams.

Topical Editor I. A. Daglis thanks A. Yau and W. K. B. Peterson for their help in evaluating this paper.

\section{References}

André, M. and Yau, A. W.: Theories and observations of ion energization and outflow in the high latitude magnetosphere, Space Sci. Rev., 80, 27-48, 1997.

André, M., Norqvist, P., Andersson, L., Eliasson, L., Eriksson, A. I., Blomberg, L., Erlandson, R. E., and Waldemark, J.: Ion energization mechanisms at $1700 \mathrm{~km}$ in the auroral region, J. Geophys. Res., 103, 4199-4222, doi:10.1029/97JA00855, 1998.

Arvelius, S., Yamauchi, M., Nilsson, H., Lundin, R., Hobara, Y., Rème, H., Bavassano-Cattaneo, M. B., Paschmann, G., Korth, A., Kistler, L. M., and Parks, G. K.: Statistics of high-altitude and high-latitude $\mathrm{O}^{+}$ion outflows observed by Cluster/CIS, Ann. Geophys., 23, 1909-1916, 2005, http://www.ann-geophys.net/23/1909/2005/.

Balogh, A., Carr, C. M., Acuña, M. H., Dunlop, M. W., Beek, T. J., Brown, P., Fornacon, K.-H., Georgescu, E., Glassmeier, K.H., Harris, J., Musmann, G., Oddy, T., and Schwingenschuh, K.: The Cluster Magnetic Field Investigation: overview of in-flight performance and initial results, Ann. Geophys., 19, 1207-1217, 2001 ,

http://www.ann-geophys.net/19/1207/2001/.

Barghouthi, I. A.: A Monte Carlo study for ion outflows at high altitude and high latitude: Barghouthi model, J. Geophys. Res. (Space Physics), 113, 8209, doi:10.1029/2008JA013274, 2008.

Bouhram, M., Malingre, M., Jasperse, J. R., Dubouloz, N., and Sauvaud, J.-A.: Modeling transverse heating and outflow of ionospheric ions from the dayside cusp/cleft. 2 Applications, Ann. Geophys., 21, 1773-1791, 2003,

http://www.ann-geophys.net/21/1773/2003/.

Bouhram, M., Klecker, B., Miyake, W., Rème, H., Sauvaud, J.-A., Malingre, M., Kistler, L., and Blăgău, A.: On the altitude dependence of transversely heated $\mathrm{O}^{+}$distributions in the cusp/cleft, Ann. Geophys., 22, 1787-1798, 2004, http://www.ann-geophys.net/22/1787/2004/.

Chang, T., Crew, G. B., Hershkowitz, N., Jasperse, J. R., Retterer, J. M., and Winningham, J. D.: Transverse acceleration of oxygen ions by electromagnetic ion cyclotron resonance with broad band left-hand polarized waves, Geophys. Res. Lett., 13, 636639, doi:10.1029/GL013i007p00636, 1986.

Chaston, C. C., Bonnell, J. W., Carlson, C. W., McFadden, J. P., Ergun, R. E., Strangeway, R. J., and Lund, E. J.: Auroral ion acceleration in dispersive Alfvén waves, J. Geophys. Res., 109, A04205, doi:10.1029/2003JA010053, 2004.

Cladis, J. B.: Parallel acceleration and transport of ions from polar ionosphere to plasmasheet, Geophys. Res. Lett., 13, 893-896, 1986.

Escoubet, C. P., Fehringer, M., and Goldstein, M.: Introduction The Cluster mission, Ann. Geophys., 19, 1197-1200, 2001, http://www.ann-geophys.net/19/1197/2001/.

Fritz, T. A., Chen, J., and Siscoe, G. L.: Energetic ions, large diamagnetic cavities, and Chapman-Ferraro cusp, J. Geophys. Res. (Space Physics), 108, 1028, doi:10.1029/2002JA009476, 2003.

Gustafsson, G., André, M., Carozzi, T., Eriksson, A. I., Fälthammar, C.-G., Grard, R., Holmgren, G., Holtet, J. A., Ivchenko, N., Karlsson, T., Khotyaintsev, Y., Klimov, S., Laakso, H., Lindqvist, P.-A., Lybekk, B., Marklund, G., Mozer, F., Mursula, K., Pedersen, A., Popielawska, B., Savin, S., Stasiewicz, K., Tanskanen, P., Vaivads, A., and Wahlund, J.-E.: First results of electric field and density observations by Cluster EFW based on initial months of operation, Ann. Geophys., 19, 1219-1240, 2001, http://www.ann-geophys.net/19/1219/2001/.

Hockney, R. W. and Eastwood, J. W.: Computer simulation using particles, Bristol, Hilger, 1988.

Lotko, W.: The magnetosphere-ionospher system from the perspective of plasma circulation: A tutorial, J. Atmos. Solar-Terr. Phys., 69, 191-211, doi:10.1016/j.jastp.2006.08.011, 2007.

Moore, T. E., Lundin, R., Alcayde, D., André, M., Ganguli, S. B., Temerin, M., and Yau, A.: Source processes in the high-altitude ionosphere, Space Sci. Rev., 88, 7-84, 1999.

Nilsson, H., Yamauchi, M., Eliasson, L., Norberg, O., and Clemmons, J.: The ionospheric signature of the cusp as seen by incoherent scatter radar, J. Geophys. Res., 101, 10947-10963, 1996.

Nilsson, H., Kirkwood, S., and Moretto, T.: Incoherent scatter radar observations of the cusp acceleration region and cusp fieldaligned currents, J. Geophys. Res., 103, 26721-26730, 1998.

Nilsson, H., Joko, S., Lundin, R., Rme, H., Sauvaud, J.-A., Dandouras, I., Balogh, A., Carr, C., Kistler, L. M., Klecker, B., Carlson, C. W., Bavassano-Cattaneo, M. B., and Korth, A.: The structure of high altitude $\mathrm{O}^{+}$energization and outflow: a case study, Ann. Geophys., 22, 2497-2506, 2004, http://www.ann-geophys.net/22/2497/2004/.

Nilsson, H., Waara, M., Arvelius, S., Marghitu, O., Bouhram, M., Hobara, Y., Yamauchi, M., Lundin, R., Rme, H., Sauvaud, J.-A., Dandouras, I., Balogh, A., Kistler, L. M., Klecker, B., Carlson, 
C. W., Bavassano-Cattaneo, M. B., and Korth, A.: Characteristics of high altitude oxygen ion energization and outflow as observed by Cluster: a statistical study, Ann. Geophys., 24, 10991112,2006 http://www.ann-geophys.net/24/1099/2006/.

Nilsson, H., Waara, M., Marghitu, O., Yamauchi, M., Lundin, R., Rème, H., Sauvaud, J.-A., Dandouras, I., Lucek, E., Kistler, L. M., Klecker, B., Carlson, C. W., Bavassano-Cattaneo, M. B., and Korth, A.: An assessment of the role of the centrifugal acceleration mechanism in high altitude polar cap oxygen ion outflow, Ann. Geophys., 26, 145-157, 2008, http://www.ann-geophys.net/26/145/2008/.

Norqvist, P., André, M., Eliasson, L., Eriksson, A. I., Blomberg, L., Lühr, H., and Clemmons, J. H.: Ion cyclotron heating in the dayside magnetosphere, J. Geophys. Res., 101, 13179-13194, doi:10.1029/95JA03596, 1996.

Norqvist, P., André, M., and Tryland, M.: A statistical study of ion energization mechanisms in the auroral region, J. Geophys. Res., 103, 23459-23474, 1998.

Rème, H., Aoustin, C., Bosqued, J. M., Dandouras, I., Lavraud, B., Sauvaud, J. A., Barthe, A., Bouyssou, J., Camus, Th., CoeurJoly, O., Cros, A., Cuvilo, J., Ducay, F., Garbarowitz, Y., Medale, J. L., Penou, E., Perrier, H., Romefort, D., Rouzaud, J., Vallat, C., Alcaydé, D., Jacquey, C., Mazelle, C., d'Uston, C., Möbius, E., Kistler, L. M., Crocker, K., Granoff, M., Mouikis, C., Popecki, M., Vosbury, M., Klecker, B., Hovestadt, D., Kucharek, H.,
Kuenneth, E., Paschmann, G., Scholer, M., Sckopke, N., Seidenschwang, E., Carlson, C. W., Curtis, D. W., Ingraham, C., Lin, R. P., McFadden, J. P., Parks, G. K., Phan, T., Formisano, V., Amata, E., Bavassano-Cattaneo, M. B., Baldetti, P., Bruno, R., Chionchio, G., Di Lellis, A., Marcucci, M. F., Pallocchia, G., Korth, A., Daly, P. W., Graeve, B., Rosenbauer, H., Vasyliunas, V., McCarthy, M., Wilber, M., Eliasson, L., Lundin, R., Olsen, S., Shelley, E. G., Fuselier, S., Ghielmetti, A. G., Lennartsson, W., Escoubet, C. P., Balsiger, H., Friedel, R., Cao, J.-B., Kovrazhkin, R. A., Papamastorakis, I., Pellat, R., Scudder, J., and Sonnerup, B.: First multispacecraft ion measurements in and near the Earth's magnetosphere with the identical Cluster ion spectrometry (CIS) experiment, Ann. Geophys., 19, 1303-1354, 2001, http://www.ann-geophys.net/19/1303/2001/.

Schunk, R. W., Raitt, W. J., and Banks, P. M.: Effect of electric fields on the daytime high-latitude $E$ and $F$ regions, J. Geophys. Res., 80, 3121-3130, 1975.

Seki, K., Elphic, R. C., Thomsen, M. F., Bonnell, J., McFadden, J. P., Lund, E. J., Hirahara, M., Terasawa, T., and Mukai, T.: A new perspective on plasma supply mechanisms to the magnetotail from a statistical comparison of dayside mirroring $\mathrm{O}^{+}$at low altitudes with lobe/mantle beams, J. Geophys. Res. (Space Physics), 107, 1047, doi:10.1029/2001JA900122, 2002.

Tsyganenko, N. A.: A magnetospheric model with a warped tail current, Planet. Space Sci., 37, 5-20, 1989.

Yau, A. W. and André, M.: Sources of ion outflow in the high latitude ionosphere, Space Sci. Rev., 80, 1-25, 1997. 